\title{
Identification, Mapping and Ethnobotany of Plant Species in the Peruvian High Andean Wetlands: Stimulating Biodiversity Conservation Efforts towards Sustainability
}

\author{
José Mostacero León ${ }^{1}$, Helmut Yabar ${ }^{2}$, Eloy López Medina ${ }^{1}$, William Zelada Estraver ${ }^{1}$, Jordán De La Cruz \\ Castillo $^{1}$ \& Armando Efraín Gil Rivero ${ }^{1}$ \\ ${ }^{1}$ Faculty of Biological Sciences, National University of Trujillo, Jr. San Martin 392, Trujillo 13007, Peru \\ ${ }^{2}$ Graduate School of Life and Environmental Sciences, University of Tsukuba, 1-1-1 Tennodai, Tsukuba, Ibaraki \\ 305-8587, Japan \\ Correspondence: Helmut Yabar, Graduate School of Life and Environmental Sciences, University of Tsukuba, 1- \\ 1-1 Tennodai, Tsukuba, Ibaraki 305-8587, Japan. Tel: 81-298-534-269. E-mail: \\ yabar.mostacero.h.ke@u.tsukuba.ac.jp
}

Received: December 1, 2020

Accepted: February 10, 2021

Online Published: February 19, 2021

doi:10.5539/jsd.v14n2p66

URL: https://doi.org/10.5539/jsd.v14n2p66

\begin{abstract}
The high Andean wetlands of Peru provide not only the well-known ecosystem services such as water storage, flood mitigation, erosion control, and purification of water, but are also a source of income for local economies (as fodder), have medicinal properties, are a source of food, contribute to the development of ecotourism among many other uses. Economic and population growth have already damaged many parts of the high Andean wetlands including their rich flora. In order to promote the conservation of its diversity and unique flora, this study conducted extensive botanical explorations to identify and map the floristic composition of the high Andean wetlands of La Libertad, Peru, as well as their influence on local communities. The authors conducted explorations taking taxonomic, biogeographic and ethno biological data of the flora species as well as their therapeutic and economic botany. The study identified 64 species of flora distributed in 46 genera and 27 families including Asteraceae (with 8 species), Juncaceae (with 7 species), Poaceae (with 6 species), Cyperaceae (with 5 species), Licopodiaceae and Rosaceae (with 4 species each), Apiaceae, Gentianaceae, Orobanchaceae and Sphagnaceae (with 3 species each) and Poligonaceae (with 2 species). With reference to economic botany, it was found that $32.8 \%$ of species constitute resources with a very good economic benefit. The study concludes that it is imperative to take actions to protect the high Andean wetlands as they are ecosystems with great biodiversity. This study contribution expects to raise concerns regarding the increasing impact of economic and population growth on the loss of not only natural habitats but species as well. Conservation efforts will help protect the heritage of the Andes wetlands for future generations.
\end{abstract}

Keywords: plant diversity, flora, ecosystem, high Andean wetlands, Peru

\section{Introduction}

Peru is among the countries that hosts the greatest biodiversity on earth accounting for the world's $10 \%$ species of flora (World Bank, 2014). The country has a variety of ecosystems with great importance both from the biological and social point of view. These ecosystems are the source of sustenance for the population and industry that have gradually increased their impact on natural resources and often in a disorganized and unplanned way (Kahn et al, 1993; Mostacero et al., 2007).

A wetland is an ecosystem flooded by water either seasonally or permanently (Keddy, 2010). A wetland is a zone generally flat or with a moderate slope, in which the surface is permanently or intermittently flooded, giving rise to a hybrid ecosystem aquatic and terrestrial, as occurs in the high Andean areas of Peru (Squeo et al., 2006; Ramsar, 2010). The benefits of wetlands can be grouped into seven basic categories: water importance; source of resources and energy; sediment retention and protection; transportation; recreation; ecotourism; and ecological importance (Tabilo, 1999). Regarding water importance, wetlands act as natural liquid retention, regulating their flow and avoiding floods. Wetlands also provide food and medicine to human populations (Tabilo, 1999; Mostacero et al., 2009; Mostacero et al., 2011). 
Due to the different types of vegetation that are usually found, wetlands contribute to the removal of nutrients by encouraging sedimentation. On the other hand, they show a recognized importance in the protection of the coastline, avoiding the erosion of estuaries and rivers. From a social and cultural point of view, wetlands are an excellent resource for recreation and tourism, due to their extraordinary landscape importance and their wide biodiversity (Dugan, 1992; Tabilo, 1999). From the ecological point of view, wetlands serve as a refuge for animals that use their environments to reproduce or feed (Kahn et al., 1993; Arana, 1998; Tabilo, 1999; Mostacero et al., 2007).

In Peru there are 16 coastal wetlands of which only four are protected within any of the categories of the National System of Protected Natural Areas (Valdivieso et al, 2016). These wetlands are located in the southern and central region of the country. The High Andean wetlands are located in the high plateaus of Ecuador, Peru, Bolivia, Argentina and Chile. While there are some conservation efforts in the region, these are limited to migratory species and wild animals (Rosetti and Stiles, 2012). In Peru there is yet to be a high Andean conservation area, despite the fact that this zone has a significant outcrop of biological richness (Arana, 1998; Kahn et al., 1993).

While the conservation of these wetlands is of great importance, the government has not given special attention to this issue. Some studies have addressed the importance of Peruvian wetlands in general. ProNaturaleza (2010) made a historic analysis of coastal wetlands in Peru and highlighted their importance and identified the main causes of their degradation including population growth, expansion of agricultural land and desertification. Ubilluz et al (2011) made a general description of a few wetlands in the central coast of Peru and identified agriculture and livestock as the main causes of their degradation. Maldonado (2014) identified the main threats to the conservation of Peruvian Andes wetlands as overgrazing, peat extraction, mining and development of infrastructure.

To the best of our knowledge there is no comprehensive plant inventory of the wetlands located in the Peruvian Andean Northern region. Moreover, the government so far hasn't introduced or implemented specific measures to protect these ecosystems. This study aims to fill this gap by identifying and safeguarding the biodiversity present in such wetlands as well as their impact on local communities. In some cases, these wetlands could constitute the only remaining habitat for some plant species and their identification is essential. The paper is organized as follows. Section 2 describes the materials and methods used during the botanical explorations, section 3 highlights the results of the explorations and discusses the results and finally, section 4 summarizes the most important findings of this study.

\section{Materials and Methods}

The present investigation was oriented to inventory the flora of the wetlands of the department of La Libertad, located between 2500 and 4500 meters above sea level (m.a.s.l.) as shown in Fig. 1. The data collection was carried out during the decade of 2008 and 2017. The botanical explorations were conducted at a rate of 4 explorations per year (summer, autumn, winter and spring); encompassing altitudinal and latitudinal transects. During the explorations, the collected botanical material and data allowed us to identify the taxonomy, habit, habitat, altitudinal distribution, form of propagation and time of flowering and fruiting. The process of herborization and taxonomic determination of the botanical material was carried out in the Herbarium Truxillense of the National University of Trujillo (HUT). 


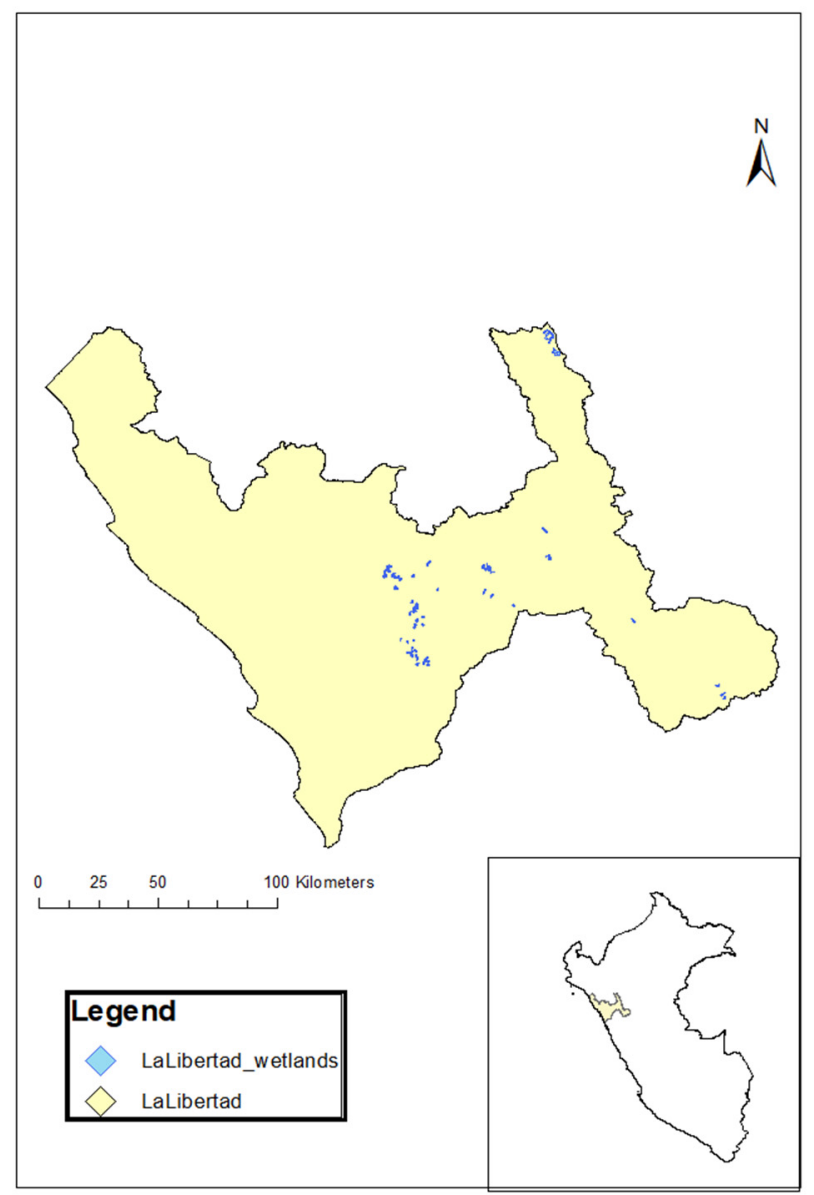

Figure 1. Map of the high Andean wetlands of La Libertad, 2017

Note: the wetlands located in La Libertad region are shown in blue. The box shows the La Libertad region location in Peru

In order to evaluate the potential benefits of the wetland species to the local people, the authors conducted a survey during the last expedition in 2017. We interviewed 384 locals (this value corresponds to the maximum sample size for a 95\% confidence level and 5\% margin of error) and asked about the value of each of the species from the following options: medicinal, dyeing, soil protector, and fodder. The results of the survey were recorded during the exploration according to the labels attached to the species registered in the HUT.

\section{Results and Discussion}

Table 1 shows the taxonomic characteristics of the flora of the high Andean wetlands. The table highlights scientific name / family, common names, habit, habitat, altitudinal distribution, propagation form, flowering time and period of fructification. 
Table 1. Characteristics of the flora of the high Andean wetlands of the Department of La Libertad, Peru

\begin{tabular}{|c|c|c|c|c|c|c|c|c|}
\hline $\mathbf{N}$ & $\begin{array}{l}\text { SCIENTIFIC NAME / } \\
\text { FAMILY }\end{array}$ & $\begin{array}{l}\text { COMMON } \\
\text { NAMES }\end{array}$ & HABIT & $\underset{(*)}{\operatorname{HABITAT}}$ & $\begin{array}{l}\text { ALTITUDE } \\
(\text { m.a.s.l. }) \\
\left({ }^{*}\right)\end{array}$ & $\begin{array}{l}\text { PROPAGATION } \\
\text { FORM }\end{array}$ & $\begin{array}{c}\text { FLOWERING } \\
\text { TIME }\end{array}$ & $\begin{array}{c}\text { TIME OF } \\
\text { FRUCTIFICATION }\end{array}$ \\
\hline 1 & $\begin{array}{l}\text { Acaena torilicarpa Bitter } \\
\text { (ROSACEAE) }\end{array}$ & - & $\begin{array}{l}\text { marsh } \\
\text { plant } 40 \\
\mathrm{~cm} \text { tall }\end{array}$ & $\begin{array}{l}\text { Disturbed } \\
\text { areas. It } \\
\text { grows in } \\
\text { wetlands, } \\
\text { preferably } \\
\text { with soil rich } \\
\text { in organic } \\
\text { matter. }\end{array}$ & $2000-4500$ & $\begin{array}{l}\text { Seeds and } \\
\text { stolons. }\end{array}$ & April-June & $\begin{array}{l}\text { June- } \\
\text { August }\end{array}$ \\
\hline 2 & $\begin{array}{ll}\text { Adiantum raddianum } & \text { C. } \\
\text { Presl (PTERIDACEAE) } & \end{array}$ & "culantrillo" & $\begin{array}{l}\text { Small } \\
\text { herb } \\
\text { riparian }\end{array}$ & $\begin{array}{l}\text { Own of flat } \\
\text { places, } \\
\text { caespitose } \\
\text { and wet. }\end{array}$ & $3000-4000$ & $\begin{array}{l}\text { Spores and } \\
\text { rhizomes. }\end{array}$ & All year & All year \\
\hline 3 & $\begin{array}{l}\text { Agrostis breviculmis Hitchc. } \\
\text { (POACEAE) }\end{array}$ & "grass". & $\begin{array}{l}\text { Prostrate } \\
\text { and } \\
\text { marsh } \\
\text { grass }\end{array}$ & $\begin{array}{c}\text { Disturbed } \\
\text { areas. It } \\
\text { grows in } \\
\text { wetlands, } \\
\text { preferably } \\
\text { with soil } \\
\text { rich in } \\
\text { organic } \\
\text { matter. }\end{array}$ & $2000-4500$ & Seeds. & All year & All year \\
\hline 4 & $\begin{array}{l}\text { Alchemilla orbiculata } \text { R. \& } \\
\text { P. (ROSACEAE) }\end{array}$ & - & $\begin{array}{l}\text { Prostrate } \\
\text { and } \\
\text { marsh } \\
\text { herb }\end{array}$ & $\begin{array}{c}\text { Ambient } \\
\text { with fog and } \\
\text { humid soils } \\
\text { rich in } \\
\text { organic } \\
\text { matter. }\end{array}$ & $2000-4500$ & $\begin{array}{l}\text { Seeds and } \\
\text { stolons. }\end{array}$ & April-June & $\begin{array}{c}\text { June- } \\
\text { August }\end{array}$ \\
\hline 5 & $\begin{array}{l}\text { Alchemilla pinnata } \text { R. \& P. } \\
\text { (ROSACEAE) }\end{array}$ & - & $\begin{array}{l}\text { Prostrate } \\
\text { and } \\
\text { marsh } \\
\text { herb }\end{array}$ & $\begin{array}{c}\text { Marsh and } \\
\text { muddy } \\
\text { slopes, } \\
\text { disturbed } \\
\text { areas. It } \\
\text { grows in } \\
\text { wetlands, } \\
\text { preferably } \\
\text { with soil } \\
\text { rich in } \\
\text { organic } \\
\text { matter. }\end{array}$ & $2000-4500$ & $\begin{array}{l}\text { Seeds and } \\
\text { stolons. }\end{array}$ & April-June & $\begin{array}{c}\text { June- } \\
\text { August }\end{array}$ \\
\hline 6 & $\begin{array}{l}\text { Alchemilla procumbrens } \\
\text { ROSE (ROSACEAE) }\end{array}$ & - & $\begin{array}{l}\text { Prostrate } \\
\text { and } \\
\text { marsh } \\
\text { herb }\end{array}$ & $\begin{array}{l}\text { Marsh and } \\
\text { muddy } \\
\text { slopes, } \\
\text { disturbed } \\
\text { areas. It } \\
\text { grows in } \\
\text { wetlands, } \\
\text { preferably } \\
\text { with soil } \\
\text { rich in } \\
\text { organic } \\
\text { matter. }\end{array}$ & $2000-4500$ & $\begin{array}{l}\text { Seeds and } \\
\text { stolons. }\end{array}$ & April-June & $\begin{array}{c}\text { June- } \\
\text { August }\end{array}$ \\
\hline 7 & $\begin{array}{lr}\begin{array}{l}\text { Baccharis } \\
\text { (Lam.) }\end{array} & \text { genistelloides } \\
\text { (ASTERACEAE) } & \text { Pers. } \\
\end{array}$ & "carqueja" & $\begin{array}{c}\text { Herb with } \\
\text { winged } \\
\text { stems }\end{array}$ & $\begin{array}{l}\text { Sub shrub of } \\
\text { marshy and } \\
\text { rocky areas. }\end{array}$ & $500-4500$ & Seeds. & May -July & July -August \\
\hline 8 & $\begin{array}{l}\text { Bartsia bartsioides (Hook.) } \\
\text { Edwin } \\
\text { (OROBANCHACEAE) }\end{array}$ & - & $\begin{array}{l}\text { Small, } \\
\text { glutinous } \\
\text { herb }\end{array}$ & $\begin{array}{l}\text { Of places } \\
\text { Palustres, } \\
\text { flooded, } \\
\text { humid and }\end{array}$ & $2000-4500$ & Seeds. & May -July & July August \\
\hline
\end{tabular}




\begin{tabular}{|c|c|c|c|c|c|c|c|c|}
\hline $\mathbf{N}$ & $\begin{array}{l}\text { SCIENTIFIC NAME / } \\
\text { FAMILY }\end{array}$ & $\begin{array}{l}\text { COMMON } \\
\text { NAMES }\end{array}$ & HABIT & $\begin{array}{l}\text { HABITAT } \\
(*)\end{array}$ & $\begin{array}{l}\text { ALTITUDE } \\
(\text { m.a.s.1.) } \\
\left({ }^{*}\right)\end{array}$ & $\begin{array}{l}\text { PROPAGATION } \\
\text { FORM }\end{array}$ & $\begin{array}{l}\text { FLOWERING } \\
\text { TIME }\end{array}$ & $\begin{array}{c}\text { TIME OF } \\
\text { FRUCTIFICATION }\end{array}$ \\
\hline \multicolumn{9}{|c|}{ flat. } \\
\hline 9 & $\begin{array}{l}\text { Bartsia tomentosa Molau } \\
\text { (OROBANCHACEAE) }\end{array}$ & - & $\begin{array}{l}\text { Small, } \\
\text { glutinous } \\
\text { herb }\end{array}$ & $\begin{array}{l}\text { Endemic to } \\
\text { the north of } \\
\text { Peru of flat, } \\
\text { swampy and } \\
\text { waterlogged } \\
\text { places. }\end{array}$ & $3000-4800$ & Seeds. & May -July & July-August \\
\hline 10 & $\begin{array}{l}\text { Bidens andicola Kunth } \\
\text { (ASTERACEAE) }\end{array}$ & " love dry " & $\begin{array}{l}\text { Small } \\
\text { herb }\end{array}$ & $\begin{array}{l}\text { Marsh and } \\
\text { swampy } \\
\text { slopes. }\end{array}$ & $3000-4500$ & Seeds. & May -July & July- October \\
\hline 11 & $\begin{array}{l}\text { Bidens squarrosa Kunth } \\
\text { (ASTERACEAE) }\end{array}$ & "love dry" & $\begin{array}{l}\text { Small } \\
\text { herb }\end{array}$ & $\begin{array}{l}\text { Marsh and } \\
\text { swampy } \\
\text { slopes }\end{array}$ & $500-3000$ & Seeds. & May -July & July- October \\
\hline 12 & $\begin{array}{l}\text { Calamagrostis } \\
\text { ligulata(Kunth) Hitchc. } \\
\text { (POACEAE) }\end{array}$ & "pajonal". & $\begin{array}{l}\text { Cespitose } \\
\text { grass }\end{array}$ & $\begin{array}{c}\text { Flooded } \\
\text { areas and } \\
\text { edge of } \\
\text { lagoons with } \\
\text { acid } \\
\text { drainages. }\end{array}$ & $3000-4600$ & Seeds. & All year & All year \\
\hline 13 & $\begin{array}{l}\text { Calamagrostis rigescens (J. } \\
\text { Presl) Scribn. (POACEAE) }\end{array}$ & "pajonal". & $\begin{array}{l}\text { Cespitose } \\
\text { grass }\end{array}$ & $\begin{array}{c}\text { Wet and } \\
\text { sub-humid } \\
\text { soils, edges } \\
\text { of rivers. }\end{array}$ & $3500-4500$ & Seeds. & All year & All year \\
\hline 14 & $\begin{array}{l}\text { Carex lemanniana } \mathrm{W} . \text { Boott } \\
\text { (CYPERACEAE) }\end{array}$ & - & $\begin{array}{l}\text { Herb of } \\
\text { stems } \\
\text { trines }\end{array}$ & $\begin{array}{l}\text { Herb of } \\
\text { regular } \\
\text { slope, } \\
\text { humid, } \\
\text { marshy and } \\
\text { aquatic. }\end{array}$ & $2500-4000$ & $\begin{array}{l}\text { Seeds and } \\
\text { rhizome }\end{array}$ & May -July & July -August \\
\hline 15 & $\begin{array}{l}\text { Castilleja arvensis Schltdl. } \\
\& \quad \text { Cham. } \\
\text { (OROBANCHACEAE) }\end{array}$ & " bull blood ". & $\begin{array}{l}\text { Herb with } \\
\text { red } \\
\text { flower }\end{array}$ & $\begin{array}{l}\text { Of flat lands } \\
\text { to moderate } \\
\text { slopes } \\
\text { humid to } \\
\text { aquatic. }\end{array}$ & $0-3800$ & Seeds. & May -July & July -August \\
\hline 16 & $\begin{array}{l}\text { Ceratophyllum demersum } \\
\text { L. } \\
\text { (CERATOPHYLLACEAE) }\end{array}$ & $\begin{array}{l}\text { "ceratofilo", } \\
\text { " fox's tail ". }\end{array}$ & Herb & $\begin{array}{l}\text { Aquatic and } \\
\text { submerged. }\end{array}$ & $10-500$ & $\begin{array}{l}\text { Seeds and } \\
\text { stolons. }\end{array}$ & All year & All year \\
\hline 17 & $\begin{array}{l}\text { Cortaderia jubata (Lem.) } \\
\text { Stapf (POACEAE) }\end{array}$ & "cortadera" & $\begin{array}{c}\text { Grass } \\
\text { with rigid } \\
\text { and } \\
\text { cutting } \\
\text { blades }\end{array}$ & $\begin{array}{c}\text { Riparian } \\
\text { grass of } \\
\text { shrub, } \\
\text { waterlogged } \\
\text { and aquatic } \\
\text { places. }\end{array}$ & $2000-4300$ & Seeds. & April -June & June -August \\
\hline 18 & $\begin{array}{l}\text { Distichia muscoides Nees \& } \\
\text { Meyen (JUNCACEAE) }\end{array}$ & "champa" & Grass & $\begin{array}{c}\text { Aquatic, } \\
\text { submerged } \\
\text { in marshy } \\
\text { and } \\
\text { waterlogged } \\
\text { soils. }\end{array}$ & $3500-4800$ & $\begin{array}{l}\text { Seeds and } \\
\text { stolons. }\end{array}$ & All year & All year \\
\hline 19 & $\begin{array}{l}\text { Epilobium denticulatum } \mathrm{R} . \\
\text { \& } \mathrm{P} .(O N A G R A C E A E)\end{array}$ & - & $\begin{array}{l}\text { Cespitose } \\
\text { herb }\end{array}$ & $\begin{array}{l}\text { Herb of } \\
\text { medium } \\
\text { slope to } \\
\text { plains, } \\
\text { humid, } \\
\text { marshy and } \\
\text { even } \\
\text { aquatic. }\end{array}$ & $3000-4500$ & Seeds. & May -July & July -August \\
\hline 20 & Equisetum bogotense Kunth & " horse tail " & Cespitose & Form & $3500-4500$ & Sorus and & All the year & All the year \\
\hline
\end{tabular}




\begin{tabular}{|c|c|c|c|c|c|c|c|c|}
\hline $\mathbf{N}$ & $\begin{array}{l}\text { SCIENTIFIC NAME / } \\
\text { FAMILY }\end{array}$ & $\begin{array}{l}\text { COMMON } \\
\text { NAMES }\end{array}$ & HABIT & $\begin{array}{l}\text { HABITAT } \\
\left(^{*}\right)\end{array}$ & $\begin{array}{l}\text { ALTITUDE } \\
(\text { m.a.s.1.) } \\
\left({ }^{*}\right)\end{array}$ & $\begin{array}{l}\text { PROPAGATION } \\
\text { FORM }\end{array}$ & $\begin{array}{l}\text { FLOWERING } \\
\text { TIME }\end{array}$ & $\begin{array}{c}\text { TIME OF } \\
\text { FRUCTIFICATION }\end{array}$ \\
\hline & (EQUISETACEAE) & & herb & $\begin{array}{l}\text { pajonales, } \\
\text { humid soil. }\end{array}$ & & rhizomes. & & \\
\hline 21 & $\begin{array}{l}\text { Festuca breviaristata Pilg. } \\
\text { (POACEAE) }\end{array}$ & "pajonal". & $\begin{array}{l}\text { Cespitose } \\
\text { grass }\end{array}$ & $\begin{array}{l}\text { Semi-humid } \\
\text { ambient } \\
\text { associated }\end{array}$ & $4100-4500$ & Seeds. & All the year & All the year \\
\hline 22 & $\begin{array}{l}\text { Festuca rigidifolia Tovar } \\
\text { (POACEAE) }\end{array}$ & "pajonal". & $\begin{array}{l}\text { Pubescent } \\
\text { grass }\end{array}$ & $\begin{array}{l}\text { River grass } \\
\text { and marsh. } \\
\text { It grows in } \\
\text { wetlands, } \\
\text { preferably } \\
\text { with soil } \\
\text { rich in } \\
\text { organic } \\
\text { matter. }\end{array}$ & $1000-4000$ & Seeds. & All the year & All the year \\
\hline 23 & $\begin{array}{l}\text { Gentianella bicolor (Wedd.) } \\
\text { Fabris ex J.S. Pringle } \\
\text { (GENTIANACEAE) }\end{array}$ & "corpus huay". & $\begin{array}{l}\text { Pubescent } \\
\text { herb }\end{array}$ & $\begin{array}{l}\text { Disturbed } \\
\text { areas. It } \\
\text { grows in } \\
\text { wetlands, } \\
\text { preferably } \\
\text { with soil } \\
\text { rich in } \\
\text { organic } \\
\text { matter. }\end{array}$ & $1000-3000$ & Seeds. & May -July & July- September \\
\hline 24 & $\begin{array}{l}\text { Gentianella chamuchui } \\
\text { (Reimers) Fabris } \\
\text { (GENTIANACEAE) }\end{array}$ & "chamuchui" & $\begin{array}{c}\text { Small } \\
\text { herb with } \\
\text { yellow } \\
\text { flowers }\end{array}$ & $\begin{array}{l}\text { Own of } \\
\text { places plans } \\
\text { or of slope } \\
\text { of little } \\
\text { slope, } \\
\text { humid, } \\
\text { swampy and } \\
\text { flooded. }\end{array}$ & $3000-3500$ & Seeds. & May -July & July-September \\
\hline 25 & $\begin{array}{l}\text { Halenia vincetoxicoides } \\
\text { Gilg (GENTIANACEAE) }\end{array}$ & - & Herb & $\begin{array}{l}\text { Of slopes, } \\
\text { swamps and } \\
\text { flooded. }\end{array}$ & $2500-4000$ & Seeds. & May -July & July- September \\
\hline 26 & $\begin{array}{l}\text { Huperzia crassa (Humb. \& } \\
\text { Bonpl. ex Willd.) Rothm. } \\
\text { (LYCOPODIACEAE) }\end{array}$ & "trencilla". & $\begin{array}{l}\text { Creeping } \\
\text { herbs - } \\
\text { ascendant }\end{array}$ & $\begin{array}{c}\text { Creeping } \\
\text { herbs from } \\
\text { places of } \\
\text { plans or } \\
\text { slopes of } \\
\text { ponca } \\
\text { pending, } \\
\text { wet, marshy } \\
\text { and flooded. }\end{array}$ & $2500-4000$ & Sorus. & All the year & All the year \\
\hline 27 & $\begin{array}{l}\text { Huperzia saururus (Lam.) } \\
\text { Trevis. } \\
\text { (LYCOPODIACEAE) }\end{array}$ & "trencilla". & $\begin{array}{l}\text { Water } \\
\text { herb }\end{array}$ & $\begin{array}{c}\text { Aquatic } \\
\text { grass grows } \\
\text { in swampy } \\
\text { and marshy } \\
\text { places. }\end{array}$ & $0-3500$ & Sorus. & All the year & All the year \\
\hline 28 & $\begin{array}{l}\text { Huperzia tetragona } \text { (Hook. } \\
\& \quad \text { Grev.) } \quad \text { Trevis. } \\
\text { (LYCOPODIACAEAE) }\end{array}$ & "órnamo" & $\begin{array}{l}\text { Water } \\
\text { herb }\end{array}$ & $\begin{array}{c}\text { Aquatic } \\
\text { grass grows } \\
\text { in swampy } \\
\text { and marshy } \\
\text { places. }\end{array}$ & $1500-3500$ & Sorus. & All the year & All the year \\
\hline 29 & $\begin{array}{l}\text { Hydrocotyle humboldtii A. } \\
\text { Richard (APIACEAE) }\end{array}$ & " Abad's hat " & $\begin{array}{c}\text { Flowering } \\
\text { bush of } \\
\text { yellow } \\
\text { flowers }\end{array}$ & $\begin{array}{l}\text { Of cloudy } \\
\text { and marshy } \\
\text { slopes or } \\
\text { very humid. }\end{array}$ & $2000-4500$ & $\begin{array}{l}\text { Seeds and } \\
\text { stolons. }\end{array}$ & May -July & July -August \\
\hline
\end{tabular}




\begin{tabular}{|c|c|c|c|c|c|c|c|c|}
\hline $\mathbf{N}$ & $\begin{array}{l}\text { SCIENTIFIC NAME / } \\
\text { FAMILY }\end{array}$ & $\begin{array}{l}\text { COMMON } \\
\text { NAMES }\end{array}$ & HABIT & $\begin{array}{l}\text { HABITAT } \\
\left({ }^{*}\right)\end{array}$ & $\begin{array}{l}\text { ALTITUDE } \\
(\text { m.a.s.1.) }\end{array}$ & $\begin{array}{l}\text { PROPAGATION } \\
\text { FORM }\end{array}$ & $\begin{array}{l}\text { FLOWERING } \\
\text { TIME }\end{array}$ & $\begin{array}{c}\text { TIME OF } \\
\text { FRUCTIFICATION }\end{array}$ \\
\hline 30 & $\begin{array}{l}\text { Hydrocotyle umbellata L. } \\
\text { (APIACEAE) }\end{array}$ & " Abad's hat " & $\begin{array}{l}\text { Cespitose } \\
\text { herb with } \\
\text { yellow } \\
\text { capitulos }\end{array}$ & $\begin{array}{l}\text { Herb of flat, } \\
\text { marshy or } \\
\text { very humid } \\
\text { places of the } \\
\text { Jalca. }\end{array}$ & $3000-3500$ & $\begin{array}{l}\text { Seeds and } \\
\text { stolons. }\end{array}$ & May -July & July -August \\
\hline 31 & $\begin{array}{l}\text { Hypericum laricifolium } \\
\text { Juss. (HYPERICACEAE) }\end{array}$ & "chinchango" & $\begin{array}{l}\text { Cespitose } \\
\text { herb with } \\
\text { yellow } \\
\text { capitulos }\end{array}$ & $\begin{array}{l}\text { Herb of flat, } \\
\text { marshy or } \\
\text { very humid } \\
\text { places of the } \\
\text { Jalca. }\end{array}$ & $1500-4800$ & Seeds. & April-June & June -August \\
\hline 32 & $\begin{array}{l}\text { Hypochaeris schizoglossa } \\
\text { Cabrera (ASTERACEAE) }\end{array}$ & - & $\begin{array}{l}\text { Marsh } \\
\text { herb }\end{array}$ & $\begin{array}{l}\text { Grass of flat, } \\
\text { marshy or } \\
\text { very humid } \\
\text { places of the } \\
\text { Jalca. }\end{array}$ & $2500-4000$ & Seeds. & April -June & June- August \\
\hline 33 & $\begin{array}{l}\text { Hypochaeris taraxacoides } \\
\text { (Walpers) Bentham \& } \\
\text { Hokker f. (ASTERACEAE) }\end{array}$ & - & $\begin{array}{c}\text { Marsh } \\
\text { herb }\end{array}$ & $\begin{array}{l}\text { Herb of flat, } \\
\text { marshy or } \\
\text { very humid } \\
\text { places of the } \\
\text { Jalca. }\end{array}$ & $0-4000$ & Seeds. & April- June & June- August \\
\hline 34 & $\begin{array}{l}\text { Juncus arcticus Willd. } \\
\text { (JUNCACEAE) }\end{array}$ & "junco" & $\begin{array}{l}\text { Marsh } \\
\text { grass }\end{array}$ & $\begin{array}{l}\text { Grass of flat, } \\
\text { marshy or } \\
\text { very humid } \\
\text { places of the } \\
\text { Jalca. }\end{array}$ & $3500-4500$ & $\begin{array}{l}\text { Seeds and } \\
\text { stolons. }\end{array}$ & April -June & June- August \\
\hline 35 & $\begin{array}{l}\text { Juncus bufonius } \\
\text { (JUNCACEAE) }\end{array}$ & "junco" & $\begin{array}{l}\text { Marsh } \\
\text { grass }\end{array}$ & $\begin{array}{l}\text { Grass of flat, } \\
\text { marshy or } \\
\text { very humid } \\
\text { places of the } \\
\text { Jalca. }\end{array}$ & $2000-4000$ & $\begin{array}{l}\text { Seeds and } \\
\text { stolons. }\end{array}$ & April -June & June- August \\
\hline 36 & $\begin{array}{l}\text { Juncus imbricatus Laharpe } \\
\text { (JUNCACEAE) }\end{array}$ & "junco" & $\begin{array}{l}\text { Marsh } \\
\text { grass }\end{array}$ & $\begin{array}{l}\text { Grass of flat, } \\
\text { marshy or } \\
\text { very humid } \\
\text { places of the } \\
\text { Jalca. }\end{array}$ & $1500-4000$ & $\begin{array}{l}\text { Seeds and } \\
\text { rhizomes. }\end{array}$ & April -June & June- August \\
\hline 37 & $\begin{array}{l}\text { Juncus pallescens Lamarck } \\
\text { (JUNCACEAE) }\end{array}$ & "junco" & Grass & $\begin{array}{c}\text { Floating } \\
\text { aquatic grass }\end{array}$ & $2000-2500$ & $\begin{array}{l}\text { Seeds and } \\
\text { rhizomes. }\end{array}$ & April -June & June- August \\
\hline 38 & $\begin{array}{l}\text { Lemna minuta } \\
\text { Kunth.(ARACEAE) }\end{array}$ & " duckweed " & $\begin{array}{c}\text { Small } \\
\text { herb with } \\
\text { blue } \\
\text { flowers }\end{array}$ & $\begin{array}{l}\text { From flat to } \\
\text { low slope, } \\
\text { wet, } \\
\text { swampy and } \\
\text { waterlogged. }\end{array}$ & $2500-3500$ & $\begin{array}{l}\text { Seeds and } \\
\text { stolons. }\end{array}$ & May -June & June- August \\
\hline 39 & $\begin{array}{l}\text { Lobelia tenera Kunth } \\
\text { (CAMPANULACEAE) }\end{array}$ & - & $\begin{array}{l}\text { Marsh } \\
\text { and } \\
\text { riparian } \\
\text { grass }\end{array}$ & $\begin{array}{l}\text { Own of flat } \\
\text { places or } \\
\text { slopes of } \\
\text { little slope, } \\
\text { very humid } \\
\text { riverside and } \\
\text { marshes. }\end{array}$ & $3000-4500$ & Seeds. & May -June & July -August \\
\hline 40 & $\begin{array}{l}\text { Lophosoria quadripinnata } \\
\text { (J.F. Gmel.) C. Chr. } \\
\text { (DICKSONIACEAE) }\end{array}$ & " fern ". & Herb & $\begin{array}{c}\text { River herb, } \\
\text { rizomatosa, } \\
\text { lacustrine } \\
\text { plains. }\end{array}$ & $3000-4000$ & $\begin{array}{l}\text { Sorus and } \\
\text { rhizomes. }\end{array}$ & All the year & All the year \\
\hline 41 & \begin{tabular}{l}
\multicolumn{3}{l}{ Loricaria ferruginea (Ruiz } \\
$\& \quad$ Pav.) $\quad$ Wedd. \\
(ASTERACEAE)
\end{tabular} & " foot of hen ". & Herb & $\begin{array}{c}\text { Poscuda } \\
\text { Decumbent } \\
\text { edge of } \\
\text { ditches, } \\
\text { lagoons and } \\
\text { wetlands. }\end{array}$ & $3500-4500$ & Seeds. & April- June & June -August \\
\hline
\end{tabular}




\begin{tabular}{|c|c|c|c|c|c|c|c|c|}
\hline $\mathbf{N}$ & $\begin{array}{l}\text { SCIENTIFIC NAME / } \\
\text { FAMILY }\end{array}$ & $\begin{array}{l}\text { COMMON } \\
\text { NAMES }\end{array}$ & HABIT & $\underset{\left({ }^{*}\right)}{\text { HABITAT }}$ & $\begin{array}{l}\text { ALTITUDE } \\
(\text { m.a.s.1.) }\end{array}$ & $\begin{array}{l}\text { PROPAGATION } \\
\text { FORM }\end{array}$ & $\begin{array}{l}\text { FLOWERING } \\
\text { TIME }\end{array}$ & $\begin{array}{c}\text { TIME OF } \\
\text { FRUCTIFICATION }\end{array}$ \\
\hline 42 & $\begin{array}{l}\text { Luzula gigantea Desvaux } \\
\text { (JUNCACEAE) }\end{array}$ & "luzula" & Grass & $\begin{array}{c}\text { Riparian } \\
\text { wetlands, } \\
\text { lagoons and } \\
\text { streams. }\end{array}$ & $2800-3800$ & Seeds. & May -July & July -August \\
\hline 43 & $\begin{array}{l}\text { Lycopodium clavatum L. } \\
\text { (LICOPODEACEAE) }\end{array}$ & "licopodio" & $\begin{array}{l}\text { Floating } \\
\text { aquatic } \\
\text { herb }\end{array}$ & $\begin{array}{l}\text { Emerging in } \\
\text { shallow } \\
\text { bodies of } \\
\text { water and on } \\
\text { the banks of } \\
\text { springs or } \\
\text { ditches. }\end{array}$ & $2500-4500$ & $\begin{array}{l}\text { Sorus and } \\
\text { rhizomes. }\end{array}$ & All the year & All the year \\
\hline 44 & $\begin{array}{l}\text { Mimulus glabratus Kunth. } \\
\text { (PHRYMACEAE) }\end{array}$ & "berro yellow". & Herb & $\begin{array}{l}\text { Aquatic } \\
\text { from } \\
\text { emerged to } \\
\text { floating. }\end{array}$ & $3000-3500$ & Seeds. & May-June & July- August \\
\hline 45 & $\begin{array}{l}\text { Myriophyllum quitense } \\
\text { Kunth } \\
\text { (HALORAGACEAE) }\end{array}$ & " fox's tail ". & $\begin{array}{l}\text { Marsh } \\
\text { herb and } \\
\text { cespitose }\end{array}$ & $\begin{array}{l}\text { Plain places } \\
\text { with a little } \\
\text { slope, } \\
\text { humid, } \\
\text { marshy and } \\
\text { flooded. }\end{array}$ & $3000-4500$ & Seeds. & May -June & July -August \\
\hline 46 & $\begin{array}{l}\text { Oxychloe andina Phil. } \\
\text { (JUNCACEAE) }\end{array}$ & - & Herb & $\begin{array}{l}\text { Own grass } \\
\text { of flat } \\
\text { places, } \\
\text { cespitose, } \\
\text { humid, } \\
\text { marshy and } \\
\text { waterlogged. }\end{array}$ & $3000-4500$ & Seeds. & May -June & July -August \\
\hline 47 & $\begin{array}{l}\text { Paranephelius uniflorus } \\
\text { Poeppig (ASTERACEAE) }\end{array}$ & - & Herb & $\begin{array}{l}\text { Aquatic } \\
\text { herb, } \\
\text { riparian, } \\
\text { emerging. }\end{array}$ & $2000-4000$ & Seeds. & April- June & June -August \\
\hline 48 & $\begin{array}{l}\text { Phyllactis rigida (Ruiz \& } \\
\text { Pav.) } \\
\text { (CAPRIFOLIACEAE) }\end{array}$ & $\begin{array}{l}\text { "valeriana } \\
\text { star". }\end{array}$ & Herb & $\begin{array}{l}\text { Water herb, } \\
\text { submerged } \\
\text { and } \\
\text { emerging. }\end{array}$ & $200-3000$ & Seeds. & May -June & June -August \\
\hline 49 & $\begin{array}{l}\text { Polygonum aviculare L. } \\
\text { (POLYGONACEAE) }\end{array}$ & "water pepper". & Herb & $\begin{array}{l}\text { Water herb, } \\
\text { submerged } \\
\text { and } \\
\text { emerging. }\end{array}$ & $2000-4500$ & $\begin{array}{l}\text { Seeds and } \\
\text { stolons. }\end{array}$ & All the year & All the year \\
\hline 50 & $\begin{array}{l}\text { Polygonum } \\
\text { hydropiperoides Michx. } \\
\text { (POLYGONACEAE) }\end{array}$ & "water pepper". & $\begin{array}{l}\text { Marsh } \\
\text { herb }\end{array}$ & $\begin{array}{c}\text { Of flat } \\
\text { places, } \\
\text { humid } \\
\text { riparian and } \\
\text { marshy. }\end{array}$ & $0-4000$ & $\begin{array}{l}\text { Seeds and } \\
\text { stolons. }\end{array}$ & All the year & All the year \\
\hline 51 & $\begin{array}{l}\text { Polytrichum commune } \\
\text { Hedw. } \\
\text { (POLYTRICHACEAE) }\end{array}$ & "moss". & Herb & $\begin{array}{l}\text { Aquatic, } \\
\text { Emerging in } \\
\text { wetlands. }\end{array}$ & $50-4000$ & Spores. & April -July & July- September \\
\hline 52 & $\begin{array}{l}\text { Potamogeton pectinatus L. } \\
\text { (POTAMOGETONACEAE) }\end{array}$ & "potamogeton". & Herb & $\begin{array}{c}\text { Aquatic, } \\
\text { Emergent } \\
\text { and } \\
\text { submerged. }\end{array}$ & 2000- 4000 & $\begin{array}{l}\text { Seeds and } \\
\text { stolons. }\end{array}$ & May- June & June -August \\
\hline 53 & \begin{tabular}{llr} 
Ranunculus & \multicolumn{2}{r}{ praemorsus } \\
Kunth & ex & DC. \\
(RANUNCULACEAE)
\end{tabular} & "centella". & $\begin{array}{l}\text { Rigid, } \\
\text { marsh } \\
\text { and } \\
\text { aquatic } \\
\text { herbs. }\end{array}$ & $\begin{array}{l}\text { Riparian, } \\
\text { marsh, } \\
\text { aquatic and } \\
\text { flooded soil. }\end{array}$ & $2500-3500$ & Seeds. & May -July & June- August \\
\hline
\end{tabular}




\begin{tabular}{|c|c|c|c|c|c|c|c|c|}
\hline $\mathbf{N}$ & $\begin{array}{l}\text { SCIENTIFIC NAME / } \\
\text { FAMILY }\end{array}$ & $\begin{array}{l}\text { COMMON } \\
\text { NAMES }\end{array}$ & HABIT & $\begin{array}{l}\text { HABITAT } \\
\left({ }^{*}\right)\end{array}$ & $\begin{array}{l}\text { ALTITUDE } \\
(\text { m.a.s.1.) }\end{array}$ & $\begin{array}{c}\text { PROPAGATION } \\
\text { FORM }\end{array}$ & $\begin{array}{l}\text { FLOWERING } \\
\text { TIME }\end{array}$ & $\begin{array}{c}\text { TIME OF } \\
\text { FRUCTIFICATION }\end{array}$ \\
\hline 54 & $\begin{array}{l}\text { Rorippa } \\
\text { nasturtiumaquaticum (L.) } \\
\text { Hayek (BRASICACEAE) }\end{array}$ & "berro". & $\begin{array}{l}\text { Rigid, } \\
\text { marsh } \\
\text { and } \\
\text { aquatic } \\
\text { herbs. }\end{array}$ & $\begin{array}{c}\text { Humid } \\
\text { ambient, and } \\
\text { when mature } \\
\text { the stems } \\
\text { harden. }\end{array}$ & $50-4200$ & Seeds. & May -June & July -August \\
\hline 55 & $\begin{array}{l}\text { Scirpus pungens } \text { M. Vahl } \\
\text { (CYPERACEAE) }\end{array}$ & "totora". & Grass & $\begin{array}{l}\text { Marsh plant, } \\
\text { aquatic and } \\
\text { emergent. }\end{array}$ & $3000-3500$ & $\begin{array}{l}\text { Seeds and } \\
\text { stolons. }\end{array}$ & April- June & June -August \\
\hline 56 & $\begin{array}{lr}\text { Scirpus californicus (C.A. } \\
\text { Mey.) } & \text { Steud. } \\
\text { (CYPERACEAE) } & \end{array}$ & "totora". & Grass & $\begin{array}{l}\text { Marsh plant, } \\
\text { aquatic and } \\
\text { emergent. }\end{array}$ & $3000-3500$ & $\begin{array}{l}\text { Seeds and } \\
\text { stolons. }\end{array}$ & April- June & June -August \\
\hline 57 & $\begin{array}{l}\text { Scirpus rigidus Boeckeler } \\
\text { (CYPERACEAE) }\end{array}$ & "totora". & Grass & $\begin{array}{l}\text { Marsh plant, } \\
\text { aquatic and } \\
\text { emergent. }\end{array}$ & $3000-3500$ & $\begin{array}{l}\text { Seeds and } \\
\text { stolons. }\end{array}$ & April- June & June -August \\
\hline 58 & $\begin{array}{l}\text { Solanum americanum Mill. } \\
\text { (SOLANACEAE) }\end{array}$ & " nightshade ". & $\begin{array}{l}\text { Aquatic } \\
\text { herbs }\end{array}$ & $\begin{array}{c}\text { Biotype of } \\
\text { wetlands of } \\
\text { the Jalca or } \\
\text { Puna. }\end{array}$ & $3500-4801$ & Seeds & April -July & June- September \\
\hline 59 & $\begin{array}{l}\text { Sphagnum magellanicum } \\
\text { Brid. (SPHAGNACEAE) }\end{array}$ & "esfagno". & Herb & $\begin{array}{l}\text { Flat, marshy } \\
\text { and flooded } \\
\text { lands of the } \\
\text { jalca or } \\
\text { puna. }\end{array}$ & $2500-4000$ & Spores. & All the year & All the year \\
\hline 60 & $\begin{array}{l}\text { Sphagnum } \\
\text { mossmannianum Müll. Hal. } \\
\text { (SPHAGNACEAE) }\end{array}$ & "esfagno". & Herb & $\begin{array}{l}\text { Flat, marshy } \\
\text { and flooded } \\
\text { lands of the } \\
\text { jalca or } \\
\text { puna. }\end{array}$ & $3000-4800$ & Spores. & All the year & All the year \\
\hline 61 & $\begin{array}{l}\text { Sphagnum subbalticum } \\
\text { Warnst. (SPHAGNACEAE) }\end{array}$ & "esfagno". & Herb & $\begin{array}{l}\text { Flat, marshy } \\
\text { and flooded } \\
\text { lands of the } \\
\text { jalca or } \\
\text { puna. }\end{array}$ & $3000-4800$ & Spores. & All the year & All the year \\
\hline 62 & $\begin{array}{l}\text { Torulinium odoratum (L.) } \\
\text { Hooper (CYPERACEAE) }\end{array}$ & - & Herb & $\begin{array}{c}\text { Riverside, } \\
\text { swampy, } \\
\text { waterlogged } \\
\text { and aquatic. }\end{array}$ & $3000-3500$ & Seeds. & May -June & July -August \\
\hline 63 & $\begin{array}{lc}\text { Verónica peregrina } & L . \\
\text { (PLANTAGINACEAE) } & \end{array}$ & "verónica". & Herb & $\begin{array}{c}\text { Marsh } \\
\text { plant, } \\
\text { riverside and } \\
\text { aquatic }\end{array}$ & 2800-3501 & Seeds. & May -June & July -August \\
\hline 64 & $\begin{array}{l}\text { Werneria nubigena Kunth } \\
\text { (ASTERACEAE) }\end{array}$ & $\begin{array}{l}\text { " onion of the } \\
\text { buzzard " }\end{array}$ & Herb & $\begin{array}{c}\text { Marsh } \\
\text { plant, } \\
\text { riverside and } \\
\text { aquatic }\end{array}$ & $3500-4500$ & Seeds. & May -June & July -August \\
\hline
\end{tabular}

Legend: The $\left(^{*}\right)$ identifies the author that the given information refers to (Brako \& Zarucchi, 1993).

Figure 2 illustrates the most representative species of flora in the study area namely A) Loricaria ferruginea, B)

Werneria nubigena, and C) Distichia muscoide. 


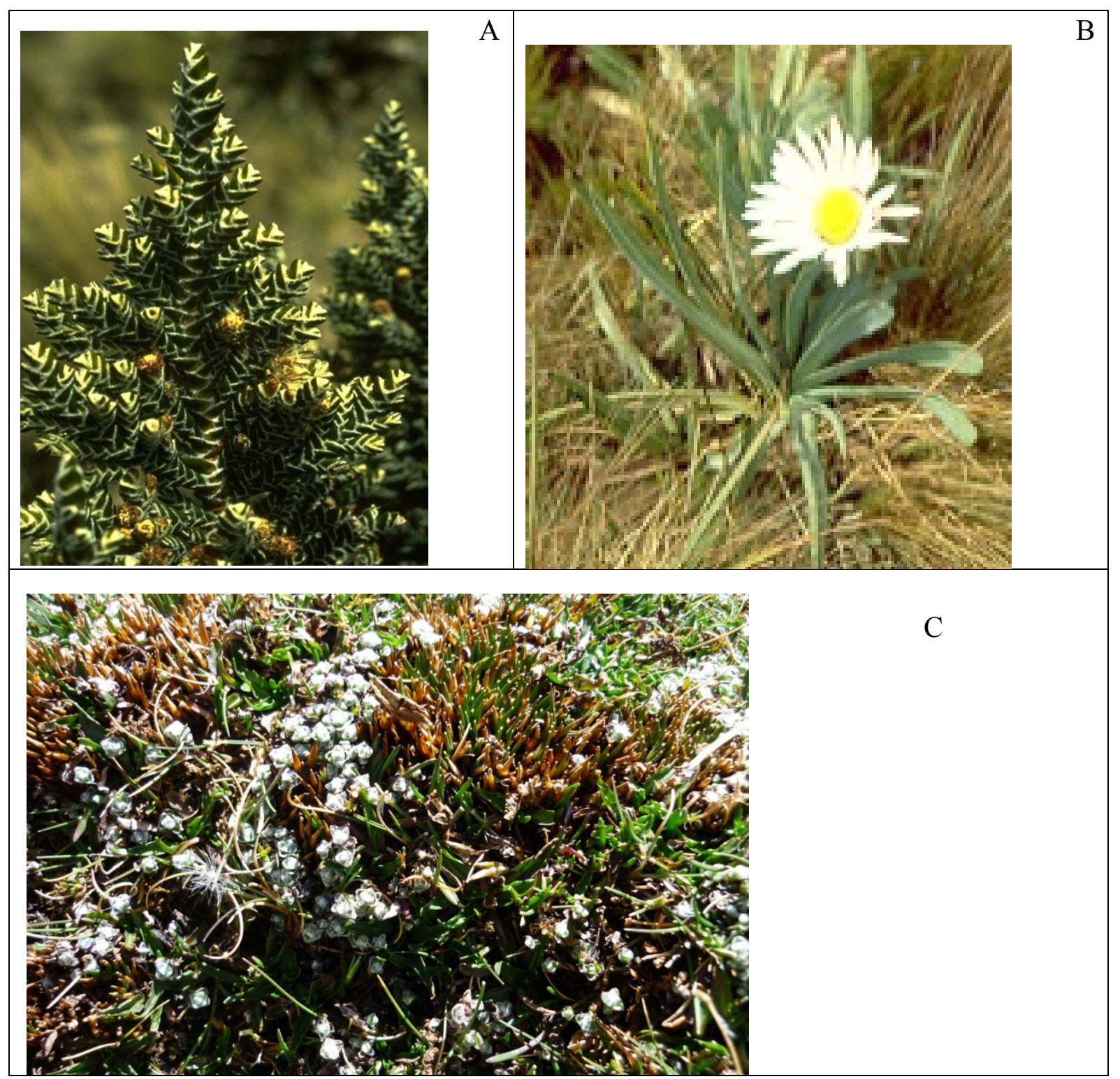

Figure 2. Representative species of flora of the high Andean wetlands of the Department of La Libertad, Peru 2008-2017

A) Loricaria ferruginea, B) Werneria nubigena, C) Distichia muscoides.

On the other hand, Figure 3, illustrates the distribution of the 64 plant species in 27 families. 


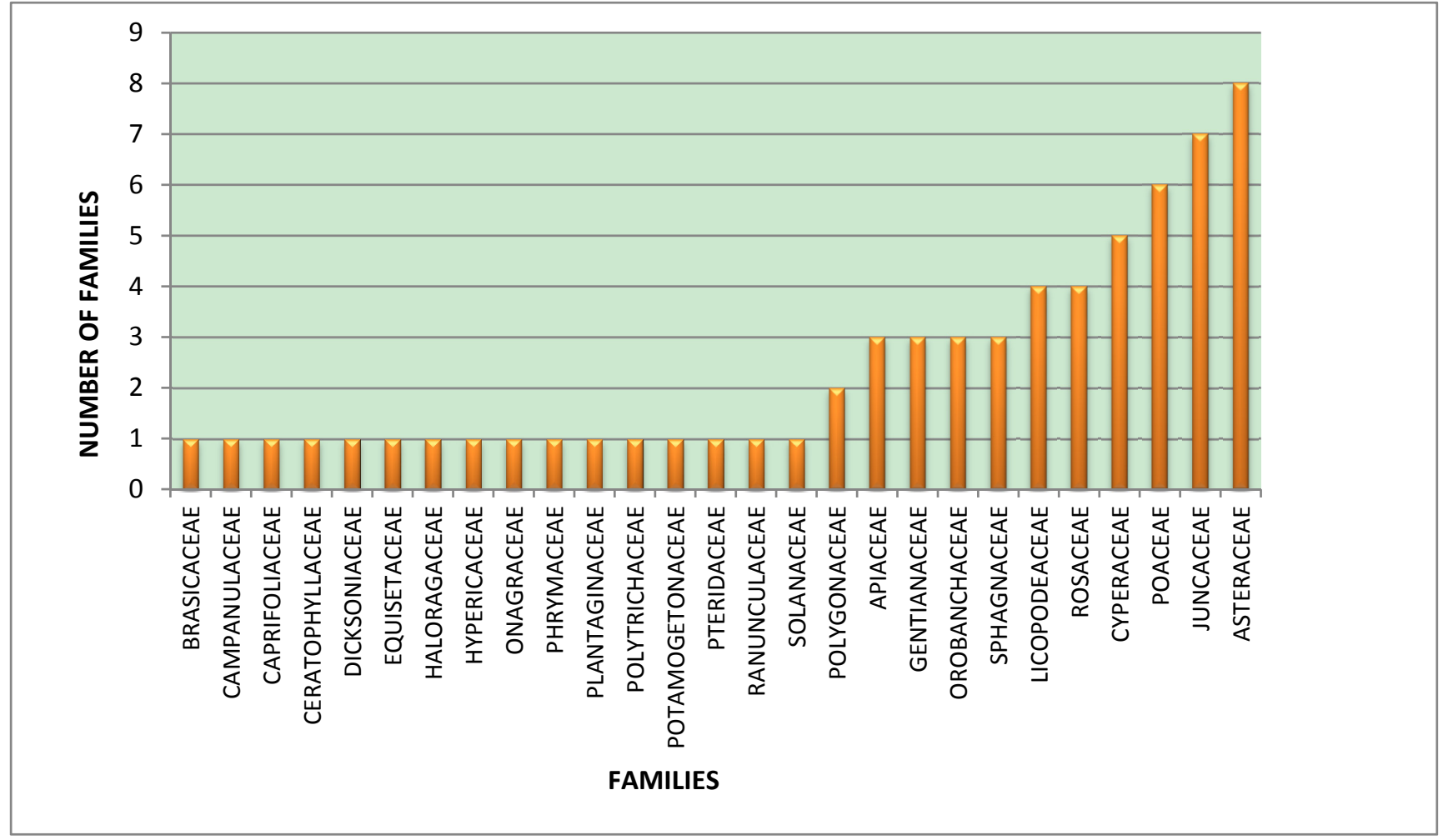

Figure 3. Distribution of the 64 species among 27 families

Table 2 and 3 present the plant species with their respective use and qualitative qualification of their benefits. From the analysis of the species we identified 13 (20.3\%) of the 64 species have fodder use, 25 (39.1\%) medicinal use, $40(62.5 \%)$ potential phytoremediation, $53(82.8 \%)$ protection of soils and $1(1.6 \%)$ dye.

Table 2. Benefits or uses of the high Andean wetland species from the La Libertad Department, Peru 2008- 2017

\begin{tabular}{|c|c|c|c|c|c|c|}
\hline \multirow[t]{3}{*}{$\mathbf{N}^{\circ}$} & \multirow[t]{3}{*}{ Species } & \multicolumn{5}{|c|}{ Socioeconomic/ Environmental Benefits } \\
\hline & & Fodder & Medicinal & Phytoremediation & Soil & Dye \\
\hline & & & & Potential & Protection & \\
\hline 1 & Acaena torilicarpa Bitter (ROSACEAE) & $\bullet$ & & $\bullet$ & $\bullet$ & \\
\hline 2 & Adiantum raddianum C. Presl (PTERIDACEAE) & & $\bullet$ & $\bullet$ & $\bullet$ & \\
\hline 3 & Agrostis breviculmis Hitchc. (POACEAE) & $\bullet$ & & $\bullet$ & $\bullet$ & \\
\hline 4 & Alchemilla orbiculata R. \& P. (ROSACEAE) & $\bullet$ & & $\bullet$ & $\bullet$ & \\
\hline 5 & Alchemilla pinnata R. \& P. (ROSACEAE) & $\bullet$ & & $\bullet$ & $\bullet$ & \\
\hline 6 & Alchemilla procumbrens ROSE (ROSACEAE) & $\bullet$ & & $\bullet$ & $\bullet$ & \\
\hline 7 & Baccharis genistelloides (Lam.) Pers. (ASTERACEAE) & $\bullet$ & $\bullet$ & & & \\
\hline 8 & Bartsia bartsioides (Hook.) Edwin (OROBANCHACEAE) & $\bullet$ & $\bullet$ & & & \\
\hline 9 & Bartsia tomentosa Molau (OROBANCHACEAE) & - & $\bullet$ & & & \\
\hline 10 & Bidens andicola Kunth (ASTERACEAE) & & $\bullet$ & & & \\
\hline 11 & Bidens squarrosa Kunth (ASTERACEAE) & & $\bullet$ & & & \\
\hline 12 & Calamagrostis ligulata(Kunth) Hitchc. (POACEAE) & $\bullet$ & & $\bullet$ & $\bullet$ & \\
\hline 13 & Calamagrostis rigescens (J. Presl) Scribn. (POACEAE) & $\bullet$ & & $\bullet$ & $\bullet$ & \\
\hline 14 & Carex lemanniana W. Boott (CYPERACEAE) & & & $\bullet$ & $\bullet$ & \\
\hline
\end{tabular}




\begin{tabular}{|c|c|c|c|c|c|c|}
\hline \multirow[t]{3}{*}{$\mathbf{N}^{\circ}$} & \multirow[t]{3}{*}{ Species } & \multicolumn{5}{|c|}{ Socioeconomic/ Environmental Benefits } \\
\hline & & \multirow[t]{2}{*}{ Fodder } & \multirow[t]{2}{*}{ Medicinal } & \multirow{2}{*}{$\begin{array}{c}\text { Phytoremediation } \\
\text { Potential }\end{array}$} & \multirow{2}{*}{$\begin{array}{c}\text { Soil } \\
\text { Protection }\end{array}$} & \multirow{2}{*}{ Dye } \\
\hline & & & & & & \\
\hline 15 & Castilleja arvensis & & $\bullet$ & & $\bullet$ & \\
\hline & (OROBANCHACEAE) & & & & & \\
\hline 16 & Cortaderia jubata (Lem.) Stapf (POACEAE) & & & & $\bullet$ & \\
\hline 17 & Distichia muscoides Nees \& Meyen (JUNCACEAE) & & & $\bullet$ & $\bullet$ & \\
\hline 18 & Epilobium denticulatum R. \& P. (ONAGRACEAE) & & & & $\bullet$ & \\
\hline 19 & Equisetum bogotense Kunth (EQUISETACEAE) & & $\bullet$ & & $\bullet$ & \\
\hline 20 & Festuca breviaristata Pilg. (POACEAE) & $\bullet$ & & $\bullet$ & $\bullet$ & \\
\hline 21 & Festuca rigidifolia Tovar (POACEAE) & $\bullet$ & & $\bullet$ & $\bullet$ & \\
\hline 22 & $\begin{array}{l}\text { Gentianella bicolor (Wedd.) Fabris ex J.S. Pringle } \\
\text { (GENTIANACEAE) }\end{array}$ & & $\bullet$ & $\bullet$ & & \\
\hline 23 & $\begin{array}{lll}\text { Gentianella chamuchui } & \text { (Reimers) } & \text { Fabris } \\
\text { (GENTIANACEAE) } & & \\
\end{array}$ & & $\bullet$ & $\bullet$ & & \\
\hline 24 & Halenia vincetoxicoides Gilg (GENTIANACEAE) & & $\bullet$ & & $\bullet$ & \\
\hline 25 & $\begin{array}{l}\text { Huperzia crassa (Humb. \& Bonpl. ex Willd.) Rothm. } \\
\text { (LYCOPODIACEAE) }\end{array}$ & & $\bullet$ & $\bullet$ & & \\
\hline 26 & Huperzia saururus (Lam.) Trevis. (LYCOPODIACEAE) & & $\bullet$ & $\bullet$ & & \\
\hline 27 & $\begin{array}{lrlll}\text { Huperzia tetragona } & \text { (Hook. } & \& & \text { Grev.) } & \text { Trevis. } \\
\text { (LYCOPODIACAEAE) } & & & & \end{array}$ & & $\bullet$ & $\bullet$ & & \\
\hline 28 & Hydrocotyle humboldtii A. Richard (APIACEAE) & & $\bullet$ & $\bullet$ & $\bullet$ & \\
\hline 29 & Hydrocotyle umbellata L. (APIACEAE) & & $\bullet$ & $\bullet$ & $\bullet$ & \\
\hline 30 & Hypericum laricifolium Juss. (HYPERICACEAE) & & & & & $\bullet$ \\
\hline 31 & Hypochaeris schizoglossa Cabrera (ASTERACEAE) & & & $\bullet$ & $\bullet$ & \\
\hline 32 & Hypochaeris taraxacoides (Walpers) Bentham \& Hokker f. & & & $\bullet$ & $\bullet$ & \\
\hline & (ASTERACEAE) & & & & & \\
\hline 33 & Juncus arcticus Willd. (JUNCACEAE) & & & $\bullet$ & $\bullet$ & \\
\hline 34 & Juncus bufonius L. (JUNCACEAE) & & & $\bullet$ & $\bullet$ & \\
\hline 35 & Juncus imbricatus Laharpe (JUNCACEAE) & & & $\bullet$ & $\bullet$ & \\
\hline 36 & Juncus microcephalus H.B.K. (JUNCACEAE) & & & $\bullet$ & $\bullet$ & \\
\hline 37 & Juncus pallescens Lamarck (JUNCACEAE) & & & $\bullet$ & $\bullet$ & \\
\hline 38 & Lemna minuta Kunth. (ARACEAE) & $\bullet$ & & $\bullet$ & $\bullet$ & \\
\hline 39 & Lobelia tenera Kunth (CAMPANULACEAE) & & $\bullet$ & & $\bullet$ & \\
\hline 40 & Lophosoria quadripinnata (J.F. Gmel.) C. Chr. & & & & & \\
\hline & (DICKSONIACEAE) & & & $\bullet$ & $\bullet$ & \\
\hline 41 & Loricaria ferruginea (Ruiz \& Pav.) Wedd. (ASTERACEAE) & & $\bullet$ & $\bullet$ & $\bullet$ & \\
\hline 42 & Luzula gigantea Desvaux (JUNCACEAE) & & & $\bullet$ & $\bullet$ & \\
\hline 43 & Lycopodium clavatum L. (LICOPODEACEAE) & & $\bullet$ & & $\bullet$ & \\
\hline 44 & Mimulus glabratus Kunth. (PHRYMACEAE) & & & $\bullet$ & $\bullet$ & \\
\hline 45 & Myriophyllum quitense Kunth (HALORAGACEAE) & & & & $\bullet$ & \\
\hline 46 & Oxychloe andina Phil. (JUNCACEAE) & & & & $\bullet$ & \\
\hline
\end{tabular}




\begin{tabular}{|c|c|c|c|c|c|c|}
\hline \multirow[t]{3}{*}{$\mathbf{N}^{\circ}$} & Species & \multicolumn{5}{|c|}{ Socioeconomic/ Environmental Benefits } \\
\hline & & Fodder & Medicinal & Phytoremediation & & Dye \\
\hline & & & & Potential & Protection & \\
\hline 47 & Paranephelius uniflorus Poeppig (ASTERACEAE) & & & & $\bullet$ & \\
\hline 48 & Phyllactis rigida (Ruiz \& Pav.) Pers. (CAPRIFOLIACEAE) & & $\bullet$ & $\bullet$ & $\bullet$ & \\
\hline 49 & Polygonum aviculare L. (POLYGONACEAE) & & & & $\bullet$ & \\
\hline 50 & Polygonum hydropiperoides Michx. (POLYGONACEAE) & & & & $\bullet$ & \\
\hline 51 & Polytrichum commune Hedw. (POLYTRICHACEAE) & & & & $\bullet$ & \\
\hline 52 & Potamogeton pectinatus L. (POTAMOGETONACEAE) & & & & $\bullet$ & \\
\hline 53 & $\begin{array}{llll}\text { Ranunculus praemorsus } & \text { Kunth ex } & \text { DC. } \\
\text { (RANUNCULACEAE) } & & & \end{array}$ & & $\bullet$ & $\bullet$ & $\bullet$ & \\
\hline 54 & $\begin{array}{ll}\text { Rorippa nasturtiumaquaticum } & \text { (L.) Hayek } \\
\text { (BRASICACEAE) } & \end{array}$ & & $\bullet$ & $\bullet$ & $\bullet$ & \\
\hline 55 & Scirpus pungens $\mathrm{M}$. Vahl (CYPERACEAE) & & & $\bullet$ & $\bullet$ & \\
\hline 56 & Scirpus californicus (C.A. Mey.) Steud. (CYPERACEAE) & & & $\bullet$ & $\bullet$ & \\
\hline 57 & Scirpus rigidus Boeckeler (CYPERACEAE) & & & $\bullet$ & $\bullet$ & \\
\hline 58 & Solanum americanum Mill. (SOLANACEAE) & & $\bullet$ & & $\bullet$ & \\
\hline 59 & Sphagnum magellanicum Brid. (SPHAGNACEAE) & & & $\bullet$ & $\bullet$ & \\
\hline 60 & Sphagnum mossmannianum Müll. Hal. (SPHAGNACEAE) & & & $\bullet$ & $\bullet$ & \\
\hline 61 & Sphagnum subbalticum Warnst. (SPHAGNACEAE) & & & $\bullet$ & $\bullet$ & \\
\hline 62 & Torulinium odoratum (L.) Hooper (CYPERACEAE) & & & & $\bullet$ & \\
\hline 63 & Verónica peregrina L. (PLANTAGINACEAE) & & $\bullet$ & & $\bullet$ & \\
\hline 64 & Werneria nubigena Kunth (ASTERACEAE) & & $\bullet$ & & $\bullet$ & \\
\hline
\end{tabular}

Table 3. Main uses of the flora of the high Andean wetlands of the Department of La Libertad, Peru 2008-2017

\begin{tabular}{llllll}
\hline Total & Fodder & Medicinal & $\begin{array}{l}\text { Phytoremediation } \\
\text { Potential }\end{array}$ & $\begin{array}{l}\text { Soil } \\
\text { Protection }\end{array}$ & Dye \\
\hline Total & 12 & 25 & 40 & 53 & 1 \\
$\%$ & 18.8 & 39.1 & 62.5 & 82.8 & 1.6 \\
\hline
\end{tabular}

In the High Andean wetlands of La Libertad it is reported the presence of 64 species of flora. The number of species in La Libertad wetlands exceeds to the Pantanos de Villa, Lima, where 47 species were reported (Ramirez and Cano, 2010) and the wetlands of Puerto Viejo, Cañete, where 32 species were reported (La Torre and Aponte, 2009). On the other hand, regarding the families with the highest number of species in the high Andean wetlands of La Libertad, were: Asteraceae (8), Juncaceae (7), Poaceae (6). Researches corroborate this information about the predominance of species of families: Poaceae and Asteraceae (Ramírez and Cano, 2010; La Torre and Aponte, 2009). By comparing the data obtained with similar works in other wetlands, we can consider the importance of these ecosystems to house an important wealth of flora.

From the analysis in Table 2, it is clear that most of the high Andean wetland species have fodder use, medicinal, and phytoremediation use, among others. Of all of them, medicinal use (39.1\%), soil protection function (82.8\%) and phytoremediation potential $(62.5 \%)$ predominate. These results are in accordance with another study that states that wetlands are sources of medicinal, forage resources, avoiding the erosion (Aponte \& Cano, 2013). On the other hand, Tables 3 highlights that $32.8 \%$ of the species can have a very good use. Studies on economic botany affirm that these ecosystems contribute economically with the surrounding communities to be a source of natural 
resources and ecotourism. On the other hand, it is important to consider that they constitute reservoirs of biological diversity, regulating and maintaining the microclimate by contributing to carbon capture (Dugan, 1990; Kahn, et al., 1993; Tabilo, 1999; Arana, 1998).

\section{Conclusions}

The high Andean wetlands of La Libertad comply with $75 \%$ of the world's environmental services (Table 4). Studies have shown that wetlands perform many vital functions, such as: water storage, flood mitigation, erosion control, purification of water by retaining sediment and pollutants and stabilization of local climatic conditions, particularly precipitation and the temperature (Tabilo, 1999; Mostacero et al., 2009; Mostacero et al., 2011). According to Barry \& Salt (2012), environmental variation and anthropogenic disturbances can influence this ecosystem. Although wetlands have a complex structure and particles, activities such as agricultural expansion and livestock are its main threats (Aponte \& Ramírez, 2011).

Table 4. Comparison of the environmental services of the wetlands of the world with those of La Libertad, Peru

\begin{tabular}{lccccccccccc}
\hline \multicolumn{1}{c}{ Wetlands } & \multicolumn{4}{c}{ Environmental services } & \multicolumn{3}{c}{ Consideration } \\
\cline { 2 - 11 } & L & NR & EI & WI & RA & R\&EC & SR \& P & MB & N $^{\circ}$ & $\%$ \\
\hline Of the world & X & X & X & X & X & X & X & X & 8 & 100 \\
High Andean of La Libertad & X & X & X & X & X & - & X & - & 6 & 75 \\
\hline
\end{tabular}

Legend: $\mathrm{EI}=$ ecological importance; $\mathrm{R} \& \mathrm{EC}=$ recreation and ecotourism; $\mathrm{L}=$ Livestock; $\mathrm{WS}=$ water storage; $\mathrm{SR}$ $\& \mathrm{P}=$ sediment retention and protection; $\mathrm{NR}=$ natural resources; $\mathrm{RA}=$ reservoir-agriculture; $\mathrm{MB}=\mathrm{Migration}$ of birds.

At present these ecosystems are undergoing significant changes caused by the rapid economic and population growth as reported in other cases (Arana, 1998; Ramirez \& Cano, 2010). As Peru doesn't have a national wetland plant inventory there is an urgent need to generate more information and develop conservation programs (Dugan, 1990; Tabilo, 1999).

In order to promote the conservation of plant diversity it is very important to have a comprehensive inventory first. The lack of a comprehensive study on the wetlands located in the Andean region of Peru coupled with the absence of specific measures to protect these ecosystems were the main drivers of this research. The study concluded that the high Andean wetlands of La Libertad, Peru, are ecosystems with great biodiversity that contribute to the conservation of at least 64 species of flora. By determining the floristic composition of the high Andean wetlands of La Libertad, Peru, as well as their impact on local communities this study aimed to raise awareness on the importance of their preservation for present and future generations.

\section{Acknowledgments}

A special thanks to the Herbarium Truxillense of the National University of Trujillo (HUT) for the taxonomic determination of the botanical material.

\section{References}

Angulo, F., Schulenberg, T., \& Edevaly, E. (2010). Birds of Eten marshes, Lambayeque, Perú. Ecol. apl., 9(2), 7181. https://doi.org/10.21704/rea.v9i1-2.397

Aponte, H., \& Cano, A. (2013). Estudio florístico comparativo de seis humedales de la costa de Lima (Perú): Actualización y nuevos retos para su conservación. Latin American Journal of Conservation, 3(2), 15-27. Retrieved from https://www.Cienti fica.edu.pe/documentos/pdfs/Aponte\&Cano_2013_RLC32_15_27.pdf

Aponte, H., \& Ramírez, D. (2011). Wetlands of the central coast of Peru: structure and menaces of its vegetal communities. Ecología Aplicada, 10(1), 31-39. https://doi.org/10.21704/rea.v10i1-2.411

Arana, C. (1998). Los pantanos de Villa: biología y conservación. Universidad Nacional Mayor de San Marcos, Museo de Historia Natural (Eds.), Lima, Perú. 238 p. Retrieved from https://www.researchgate.net/publication/288653512_Relaciones_fitogeograficas_de_la_flora_vascular_de _los_Pantanos_de_Villa

Barry, W., \& Salt, D. (2012). Resilience Practice: Building Capacity to Absorb Disturbance and Maintain Function. Island Press (eds.), Washington. 240 p. $\quad$ Retrieved from https://books.google.com.pe/books?id=LAEBev5Ljr8C\&dq=Building+Capacity+to+Absorb+Disturbance + 
and+Maintain+Function.+Washington:+Island.\&hl=es\&source $=$ gbs_navlinks_s

Brako, L., \& Zarucchi, J. (1993). Catalogue of the Flowering Plants and Gymnosperms in Peru. Monogr. Syst. Bot. Missouri Bot. Gard. Retrieved from https://www. researchgate.net/publication/270769424_Catalogue_of_the_Flowering_Plants_and_Gymnosperms_of_Peru

Dugan, P. (1990). Wetland Conservation: A Review of Current Issues and Required Action. International Union for Conservation of Nature and Natural Resources. Gland, Switzerland. Retrieved from https://books.google.com.pe/books?id=Ra

raYlXy_SsC\&dq $=$ Wetland + Conservation: $+\mathrm{A}+$ Review + of + Current + Issues + and + Required + Action.\&hl $=$ es \&source $=$ gbs_navlinks_s

Dugan, P. (1992). Conservación de Humedales, un análisis de temas de actualidad y acciones necesarias. Unión Mundial para la Naturaleza (Eds.), Gland, Suiza. 100 p. Retrieved from https://books.google.com.pe/books?id=RLq2HzktBX4C\&dq=Conservaci\%C3\%B3n+de+Humedales, + un $+\mathrm{a}$ $\mathrm{n} \% \mathrm{C} 3 \%$ Allisis $+\mathrm{de}+$ temas $+\mathrm{de}+$ actualidad $+\mathrm{y}+$ acciones + necesarias. + Suiza.\&hl $=$ es\&source $=$ gbs_navlinks_s

Kahn, F., León, B., \& Young, K. (1993). Las Plantas Vasculares en las Aguas Continentales del Perú. Instituto Francés de Estudios Andinos (IFEA). Tomo 75, Lima, Perú. 357 p. Retrieved from $\mathrm{http} / /$ horizon.documentation.ird.fr/exl-doc/pleins_text es/divers09-06/39107.pdf

Keddy, P. A. (2010). Wetland ecology: principles and conservation (2nd ed.). New York: Cambridge University Press. https://doi.org/10.1017/CBO9780511778179

La Torre, M., \& Aponte, H. (2009). Vascular flora and vegetation from Puerto Viejo wetland. Rev. peru. biol., 16(2), 215-217. Retrieved from http://revistasinvestigacio n.unmsm.edu.pe/index.php/rpb/article/view/58

Maldonado Fonken, M. S. (2014). An introduction to the bofedales of the Peruvian High Andes. Mires and Peat, 15(4), 1-13.

Mostacero, J., Castillo, F., Mejía, F., Gamarra, O., Charcape, J., \& Ramírez, R. (2011). Plantas Medicinales del Perú: Taxonomía, Ecogeografia, Fenología y Etnobotánica. Trujillo- Perú: Asamblea Nacional de Rectores Fondo (Eds.), Lima, Perú. Retrieved from http://www.librosperuanos.com/libros/detalle/13702/Plantasmedicinales-del-Peru.-Taxonomia-Ecogeografia-Fenologia-y-Etnobotanica

Mostacero, J., Mejía, F., Zelada, W., \& Medina, C. (2007). Biogeografia del Perú. Asamblea Nacional de Rectores (Eds.), Trujillo- Perú. Retrieved from http://www.Librosperu anos.com/libros/detalle/13698/Biogeografiadel-Peru

Mostacero, L., Mejia, F., \& Gamarra, T. (2009). Fanerogamas del Perú. GRAFICART (Eds.). Trujillo, Perú.1331p. Retrieved from https://books.google.com.pe/books /about /Faner\% C3\%B 3gamas _del_Per \%C3\%BA. $\mathrm{html}$ ? id $=$ rrB qtwAACAAJ \&red ir_esc $=\mathrm{y}$

ProNaturaleza. (2010). Documento Base Para la Elaboración de una Estrategia de Conservación de los Humedales de la Costa Peruana. Conservación Internacional y RAMSAR.

Ramirez, D., \& Cano, A. (2010). State of vascular flora diversity from Pantanos de Villa (Lima - Peru). Rev. peru. biol., 17(1), 111-114. https://doi.org/10.15381/rpb.v17i1.58

Ramsar. (2010). Inventario de humedales. Secretaría de la Convención de Ramsar (Eds.), $4^{\mathrm{a}}$ edición, vol. 15. Gland, Suiza. Retrieved from https://www.ramsar.org/si tes/default/files/documents/pdf/lib/hbk4-15sp.pdf

Rosselli, L., \& Stiles, F. (2012). Local and Landscape Environmental Factors are Important for the Conservation of Endangered Wetland Birds in a High Andean Plateau. Waterbirds, 35, 453-469. https://doi.org/10.1675/063.035.0310

Squeo, F., Warner, B., Aravena, R., \& Espinoza, D. (2006). Bofedales: High altitude peatlands of the central Andes. Revista Chilena de Historia Natural, 79, 245-255. https://doi.org/10.4067/S0716-078X2006000200010

Tabilo, V. (1999). El beneficio de los humedales en América Central: El potencial de los humedales para el desarrollo. Universidad Nacional Heredia (Eds.), San José, Costa Rica. 58 p. Retrieved from https://www.researchgate.net/publication/285940037_BENEFICIO_DE_LOS_HUMEDALES_EN_AMERI CA_CENTRAL

Tabilo-Valdivieso, E., \& Burmeister, J., Chavez-Villavicencio, C., \& Zöckler, C. (2016). Humedales y aves migratorias en la costa árida del Pacífico Sudamericano. Etapa I: Evaluación Ecológica Rápida.

Ubillús, H., \& Ramírez, D. (2011). Wetlands of the central coast of Peru: structure and menaces of its vegetal 
communities. Ecol. apl., 10(1).

World Bank. (2014). Peru - Participatory Management of Protected Areas. Washington, DC: World Bank Group.

\section{Copyrights}

Copyright for this article is retained by the author(s), with first publication rights granted to the journal.

This is an open-access article distributed under the terms and conditions of the Creative Commons Attribution license (http://creativecommons.org/licenses/by/4.0/). 